\title{
Rendimiento cognitivo de personas mayores que participan en grupos organizados en la provincia de San José
}

\author{
Cognitive performance of elder people participating \\ in elder's organized groups in San José
}

\author{
María Dolores Castro Rojas ${ }^{1}$ \\ Fundación Omar Dengo, Costa Rica
}

\author{
Mónica Salazar Villanea ${ }^{2}$ \\ Universidad de Costa Rica, Costa Rica
}

Resumen. Dado que el rendimiento cognitivo es uno de los principales determinantes para el envejecimiento saludable, se realizó una primera descripción del rendimiento en funciones ejecutivas y memoria de 110 Personas Adultas Mayores (PAM) que participan en programas formales en San José, Costa Rica. Se registraron datos sociodemográficos y se evaluaron las variables de rendimiento cognitivo y nivel de participación social en grupos organizados. Al finalizar, las PAM evidenciaron un rendimiento cognitivo esperable para su edad y nivel educativo en las pruebas globales, mientras en las que evalúan funcionamiento ejecutivo y memoria mostraron un rendimiento, esperable normativamente, pero ubicado en los percentiles medios e inferiores de calificación. Pese a que las PAM no muestran un alto nivel de rendimiento cognitivo se mantienen activas socialmente en diversas actividades.

Palabras clave. Rendimiento cognitivo, participación social, envejecimiento, estilo de vida activo, funciones ejecutivas, memoria.

Abstract. Since cognitive performance is a major determinant for the development of healthy aging processes and quality of life, the profiles of cognitive performance in executive functions and memory of 110 elders participating in organized groups in Costa Rica were described. Sociodemographic data were registered, and variables of cognitive performance and level of involvement in activities were evaluated. Participant showed a cognitive performance expected for their age and educational level in screening tests for global intellectual performance whereas in tests assessing executive functioning and memory performance showed a normatively expected score; however, it was located in the middle and lower ranks. Although the participants did not show a high level of cognitive performance, they have an active lifestyle with a variety of formal activities and social participation.

Keywords. Cognitive performance, social participation, aging, active lifestyle, executive functions, memory.

\footnotetext{
${ }^{1}$ María Dolores Castro Rojas. Fundación Omar Dengo, San José, Costa Rica. Dirección postal: 1032-2050. E-mail: maria.castrorojas@ucr.ac.cr

${ }^{2}$ Mónica Salazar Villanea. Instituto de Investigaciones Psicológicas,Universidad de Costa Rica. E-mail: monica.salazarvillanea@ucr.ac.cr
} 


\section{Introducción}

El envejecimiento de la población es uno de los cambios demográficos más acelerados que demanda la adecuación de las políticas públicas para garantizar condiciones óptimas de desarrollo y participación social para nuevos sectores de población de edad avanzada. El I informe de situación de la Persona Adulta Mayor en Costa Rica (Fernández \& Robles, 2008) afirma que ese grupo experimentará un crecimiento explosivo e inédito en los próximos años.

A mitad del año 2008 habían en el país aproximadamente 278000 personas mayores de 65 años las cuales constituían el 6\% de la población costarricense, para el año 2011 ese grupo de personas representaba el 7.3\% de la población total (INEC, 2012); y se espera que en los próximos 15 ó 20 años esta cifra pase a más de 600.000 personas mayores, lo que equivale a pasar de un $7.3 \%$ a un $11.5 \%$ de la población del país. Asimismo, la población de personas de 80 años y más se duplicará y pasará de 61000 a 121 000 (Fernández \& Robles, 2008).

El cambio demográfico hace prever un aumento en la demanda de servicios dirigidos a promover y facilitar una mejor adaptación a la etapa de vejez y una mayor calidad de vida de las personas mayores. Para favorecer el diseño de ese tipo de servicios se deben considerar tanto las particularidades de este grupo poblacional, como la variabilidad interindividual en las PAM, reconociendo que los recursos personales como el rendimiento cognitivo en funciones ejecutivas y memoria, asociados a la participación social y el estilo de vida activos, son factores claves para lograr procesos de envejecimiento saludable y con calidad de vida.

El envejecimiento activo o saludable puede ser definido como un proceso adaptativo a través del ciclo vital con el fin de mantener un funcionamiento físico (incluyendo la salud) y psicológico (motor, cognición, emoción y motivación) óptimo y también altos niveles de participación social (Fernández-Ballesteros, Molina, Schettini \& Del Rey, 2012; OMS, 2002; Rowe \& Khan, 1997).

En ese sentido, se sabe que el funcionamiento cognitivo es uno de los aspectos determinantes que se relaciona con las posibilidades de adaptación en la etapa de vejez (Baltes \& Baltes, 1993; Fernández-Ballesteros, Caprara \& García, 2005; Rowe \& Kahn, 1997). Asimismo, las actividades complejas de la vida diaria requieren una variedad de habilidades cognitivas del funcionamiento ejecutivo como anticipación, selección de metas, monitoreo y secuenciación (Hultsch, Small, Hertzog \& Dixon, 1999; Plehn, Marcopulos \& McLain, 2004; Schooler \& Mulatu, 2001; Smith \& Kosslyn, 2008; Smits, Van Rijsselt, Jonker \& Hardy, 1995).

Por su parte, la conducta social también requiere que los individuos estén suficientemente organizados para iniciar y mantener contacto con otros (Plehn et al., 2004; Smith y Kosslyn, 2008; Smits et al., 1995), destacando la memoria como una de las habilidades cognitivas claves asociadas al funcionamiento social (Plehn et al., 2004).

De esta manera, se sabe que en el envejecimiento normativo ocurre un declive cognitivo general y existen diferencias más marcadas en tareas relacionadas con el funcionamiento ejecutivo tales como la memoria de trabajo, atención, velocidad en el procesamiento de nueva información y esas diferencias son mayores conforme avanza la edad (Fernández-Ballesteros el al., 2005; Lowe \& Rabitt, 2005); esas pérdidas pueden ser compensadas por un incremento en el conocimiento y la experiencia (OMS, 2002) y con estrategias tales como la participación en grupos sociales y en actividades educativas que posibiliten entrenar habilidades cognitivas (Fernández-Ballesteros et al., 2012).

Este trabajo busca contribuir a un mayor conocimiento de las características específicas de las PAM en el país respecto a su rendimiento cognitivo en las funciones ejecutivas y la memoria, con el fin de brindar aportes iniciales para contribuir a que en el futuro, las intervenciones y servicios destinados a esa población concuerden con sus capacidades cognitivas específicas; para mantenerlas u optimizarlas según sea el caso, favoreciendo la autonomía y previniendo la dependencia. También es importante considerar que existen premisas tradicionales del enfoque de envejecimiento activo o exitoso que privilegian la ausencia de enfermedad y la productividad como determinantes fundamentales 
de procesos de envejecimiento con éxito. En Costa Rica, sin embargo Salazar y Blanco (2010) incluyen también dentro de los criterios para envejecer de manera adaptiva y satisfactoria los procesos subjetivos o de elaboración interna, tales como el logro de la tarea de la gerotrascendencia. Blanco (2007) identificó que en población costarricense además del rendimiento cognitivo, variables subjetivas como la autoeficacia, el autocontrol y la satisfacción con la vida resultan los mejores predictores de procesos de envejecimiento saludable, activo o exitoso; entendidos como procesos en los cuales las personas mayores experimentan estados afectivos positivos, cuentan con redes sociales de apoyo y participan de manera regular en procesos grupales.

Por su parte Lander, (2005) menciona la satisfacción personal, la habilidad para adaptarse a los cambios a través del tiempo, el mantenimiento de un sentido de pertenencia y una vida significativa y con propósito como criterios para envejecer exitosamente; destacando que la habilidad para adaptarse, lograr metas personales valiosas, desarrollar una amplía perspectiva sobre sí mismos, los otros y el mundo, podrían ser aspectos importantes en una nueva visión multidimensional del envejecimiento exitoso. Así pues, en esta línea los resultados del presente acercamiento pretenden aportar para profundizar en el reconocimiento de la diversidad en las formas de envejecer y en el conocimiento de las características cognitivas particulares de la muestra de estudio. Para ello, se trabajó con una muestra de personas que pertenecen a la Asociación Gerontológica Costarricense (AGECO), una de las principales instituciones en el país en ofrecer opciones de participación social a las PAM.

\section{Método}

\section{Estrategia general}

Se trató de un estudio descriptivo con una única medición en el tiempo de las variables de interés (Hernández, Fernández \& Baptista, 2001). Para las variables en estudio se consideraron las siguientes dimensiones:

Rendimiento cognitivo en funciones ejecutivas. Se evaluó atención ejecutiva, inhibición de respuestas automáticas, interferencias en la atención y secuenciación.
Rendimiento cognitivo en memoria. Se trabajó con procesos de codificación, reconstrucción y reconocimiento en memoria a largo plazo y en curvas de aprendizaje en memoria de corto plazo.

Nivel de participación. Tipo, frecuencia (en horas anuales) y complejidad percibida de las actividades que realizan las personas mayores en los grupos organizados. Además se registraron las variables sociodemográficas: edad, sexo, años y nivel de escolaridad, estado civil y situación socioeconómica, así como el estado de ánimo como elementos adicionales para el análisis estadístico.

En la recolección de datos se utilizaron instrumentos de uso frecuente en la investigación internacional para la medición del rendimiento cognitivo de las personas mayores y para la medición de la variable participación se elaboró un cuestionario específico que evalúa la participación en grupos organizados de personas mayores. Una vez recolectada la información, se creó una base de datos en el programa estadístico SPSS 19. 0 y se realizaron los procedimientos de análisis estadísticos descriptivos.

\section{Participantes}

Participaron 110 PAM voluntarias que cumplían con los criterios de inclusión: tener 60 o más años; saber leer y escribir; no poseer ningún tipo de alteración psiquiátrica, neurológica y/o neuropsicólogica clínicamente demostrable y no presentar deterioro cognitivo.

\section{Instrumentos}

Las pruebas utilizadas son reportadas en la investigación a nivel internacional y en los antecedentes nacionales. Sin embargo, no se encuentran adaptadas al contexto costarricense, por lo que se aportan datos iniciales para el estudio de la confiabilidad y validez de las medidas con población nacional.

Como prueba de cribaje de capacidades cognitivas se utilizó el Mini Mental State Examination (MMSE) prueba elaborada por Folstein y colaboradores en 1975, adaptación española de Lobo, Ezquerra, Gómez, Sala y Seva (1979), que evalúa el rendimiento en orientación, memoria inmediata, atención y cálculo, recuerdo y lenguaje y praxis constructiva. Dado que el criterio 
de inclusión fue presentar un rendimiento cognitivo normal, hubo poca variabilidad en la muestra y no fue posible calcular el coeficiente Alpha de Cronbach.

En una fase anterior de investigación se realizó una prueba piloto de esta prueba con 93 PAM institucionalizados (34.4\% varones y $65.6 \%$ mujeres) con una media de edad de $M=78.34$ años, $D E=8.75$. El nivel escolar en años estuvo en un rango de 0 a 24 años, $M=8.12$ años, $D E=5.20$. En esa oportunidad la prueba total presentó un Alfa de Cronbach de .72 y todas las correlaciones ítem - total superaron el .30. Los resultados para ambas muestras apuntaron a que si bien es cierto el MMSE es útil como una prueba para discriminar entre sujetos que presentan deterioro cognitivo y sujetos sin deterioro cognitivo, no resulta útil para discriminar variabilidad en los rendimientos entre sujetos sin deterioro cognitivo. En concordancia con la literatura previa (Mitrushina \& Satz, 1991; Verna, Sloan, \& Guse, 2000; Brugnolo, Nobili, Barbieri, Dessi, Ferro, Girtler et al. 2009) se recomienda su uso como prueba de tamizaje y al utilizarlo en población general debe acompañarse de otras medidas neuropsicológicas más específicas, tal y como se hizo en el presente estudio.

Para medir el nivel de rendimiento cognitivo en funciones ejecutivas y memoria se aplicaron las siguientes pruebas y escalas:

Test del Reloj (versión en castellano de Cacho-Gutiérrez, García-García, Arcaya-Navarro, Vicente-Villardón, \& Lantada-Puebla 1999): valora el estado cognitivo en dos momentos, a la orden y a la copia. En este test puntuaciones mayores indican un deterioro menor.

Test de Stroop de colores y palabras (versión normalizada en castellano de Golden, 1994): Mide la capacidad del sujeto para separar los estímulos de nombrar colores y palabras, suprimiendo la respuesta de lectura. En este test puntuaciones mayores indican un deterioro menor.

Trail Making A y B, conocido en castellano como Test de hacer caminos (Versión en español de PeñaCasanova, Quiñones-Úbeda, Quintana-Aparicio, Aguilar, Badenes, Molinuevo, et al. 2009): Mide velocidad para la atención, secuenciación, búsqueda visual, flexibilidad mental y funcionamiento motor.

CERAD Consorcio para Establecer un Registro para la Enfermedad de Alzheimer (adaptación propia de Morris, Heyman, Mohs, Hughes, Van Belle, Fillenbaum, et al., 1989): se administraron los ejercicios correspondientes a memoria de una lista de palabras; recuerdo de una lista de palabras, reconocimiento de lista de palabras, fluidez verbal, dígitos directos y dígitos inversos.

En el caso de las pruebas Test del reloj, Test de Stroop de colores y palabras, Trail Making Test A y B y las subescalas del CERAD, por tratarse de pruebas de ejecución en las cuales se registra el desempeño y el tiempo de respuesta, no fue posible realizar el cálculo del índice Alpha de Cronbach.

Subprueba CAMCOG del CAMDEX (The Cambridge Mental Disorders of Ederly Examination) adaptación española de Llinás, Villalta y López-Pousa, (1991) se utilizaron las escalas de orientación temporal y espacial, comprensión de lenguaje, memoria remota, memoria reciente, memoria de aprendizaje, atención y cálculo y función ejecutiva. Para la subprueba total se obtuvo un $\alpha$ de Cronbach de .82 .

Se observaron evidencias de validez convergente al correlacionar las medidas cognitivas utilizadas. Entre algunas de las correlaciones encontradas se pueden mencionar $r=.46, p<.01$ entre el MMSE que evalúa el rendimiento o estado cognitivo general en las dimensiones orientación, memoria inmediata, atención y cálculo, recuerdo y lenguaje y praxis constructiva, y el Test del reloj a la copia que evalúa el estado cognitivo general: ambas, pruebas de screening del estado cognitivo general. El MMSE también correlaciona positivamente con la escala CERAD recuerdo de una lista de palabras, $(r=.42, p<.01)$, lo que indica la coincidencia entre las dimensiones memoria que evalúan el MMSE y las que evalúa la escala CERAD.

También se observaron correlaciones positivas entre el Test del reloj a la orden que evalúa el rendimiento cognitivo general y las subescalas del 
CERAD "reconocimiento de una lista de palabras" $r=.327, p<0.01$ y "dígitos inversos" que evalúan el rendimiento en memoria de trabajo $r=.3267, p<0.01$. Estos resultados muestran las correlaciones positivas esperadas entre aquellas pruebas que miden los mismos subprocesos de los constructos cognitivos.

Para medir el nivel de participación de las PAM, se elaboró un formulario que registraba el tipo de actividades, la frecuencia de las actividades en horas anuales y el nivel de complejidad percibida de las actividades realizadas. Se consideró el tipo de actividades que realizan los grupos de AGECO (AGECO, 2008) y las clasificaciones hechas en investigaciones previas, Hultsch et al. (1999) y Schooler y Mulatu, (2001). Así, el instrumento para participación incluyó 8 categorías de actividades: recreativas, sociales, de adquisición de habilidades, artísticas, físicas, de ocio cognitivo, educativas y de coordinación y dirección de grupos.

La frecuencia de las actividades se registró con una escala que incluye el número de veces que se realiza una actividad en un periodo semanal, mensual y anual y la cantidad de horas invertidas. Posteriormente, se calculó un índice de horas anuales para cada tipo de actividad. La complejidad de cada tipo de actividad se evaluó con una escala de 1 a 3 , para esta clasificación se siguieron los criterios de Schooler y Mulatu, (2001).

Este instrumento se valoró con 20 PAM (distintas a los sujetos de estudio) que participaban en grupos organizados de AGECO, para verificar que las instrucciones y la información solicitada fueran claras y de fácil respuesta para los y las participantes. Se realizó una única sesión grupal en la cual se expuso la finalidad del instrumento así como las instrucciones y el formato de respuesta y se solicitó a las PAM brindar realimentación para lograr mayor claridad en las consignas; quienes participaron en la valoración informaron que estas fueron sencillas y fáciles de comprender. Por tratarse de una prueba que mide frecuencia de actividades no se realizaron pruebas de confiabilidad.

\section{Procedimiento}

En el momento en que las PAM accedieron a participar en la investigación se procedió con la lectura y firma del consentimiento informado. Posteriormente, se aplicaron de manera individual los instrumentos en las instalaciones de AGECO, en un espacio físico privado, silencioso y con mobiliario apropiado para la tarea. La aplicación se realizó en una única sesión de aproximadamente 2.5 horas con algunos intervalos de descanso si la persona participante lo solicitaba.

\section{Análisis de datos}

Una vez determinadas la estructura y la consistencia interna de las escalas, se describieron las variables a nivel univariado; además se realizó un análisis comparativo por sexo, del desempeño de las PAM en las distintas pruebas de rendimiento cognitivo. En este caso, para las medidas de dicha variable que cumplían con la condición de distribución normal verificada mediante la prueba de normalidad Kolmogorov-Smirnov, se realizaron análisis paramétricos y cuando ese principio se incumplió se realizaron análisis no paramétricos, se trabajó con pruebas t y U de Mann Whitney, para muestras independientes.

Finalmente se realizó un análisis de correlaciones entre el rendimiento en las pruebas cognitivas y la edad de los sujetos participantes.

\section{Resultados}

\section{Descripción general de las personas participantes}

La media de edad de las PAM participantes fue de $M$ $=71.92$ años, $D E=7.41$. El $65.5 \%$ fueron mujeres y el 34.5\% varones; la mayor representación femenina en la muestra, coincide con lo señalado por Fernández y Robles (2008) en cuanto a la participación en grupos formales de PAM y con la realidad de los programas de AGECO, en los cuales en el año 2008, el 87\% de la población activa a nivel nacional correspondía a mujeres.

El 98.2\% de la muestra provenía de zonas urbanas, el $38.2 \%$ estaban casados, el $37.2 \%$ era viudo/a, el 
$10 \%$ divorciado/a y el restante $7.3 \%$ soltero o soltera. Un $83.6 \%$ de los participantes estaban jubilados y el $77 \%$ recibía una pensión de los sistemas públicos o privados que existen en el país, mientras el $23 \%$ no pertenecía a ningún sistema de pensión. El ingreso promedio mensual de los participantes se ubicaba entre 200.000 y 399.000 colones (entre 392 y 782 dólares estadounidenses), lo cual no representa ingresos particularmente altos.

El $80 \%$ de la muestra estuvo constituido por PAM entre los 60 y 79 años y únicamente el 20\% lo constituyeron personas de 80 a 87 años. El 44\% de las personas participantes contaban con algún estudio de nivel primario, seguido por el $31 \%$ con algún estudio secundario y el 30\% con algún estudio universitario. Es decir, el 70\% no cuenta con estudios a nivel universitario, por lo que se trata de personas con baja o media escolaridad y, en pocos casos, se trata de personas con formación universitaria. Esta información aunada al ingreso medio reportado caracterizan a las personas participantes como personas con bajos ingresos y baja escolaridad.

Por otra parte, se observa que ninguna de las personas que participó en el estudio vive sola, aunque quienes viven con ellos y ellas no sean sus hijos o familiares directos. Esto podría indicar que las personas participantes cuentan al menos una persona que pueda colaborarles y apoyarles si fuera necesario. En este caso el apoyo recibido estaría más relacionado con aspectos socioemocionales, pues todas las PAM participantes son personas funcionalmente independientes.

Descripción del nivel de participación en grupos organizados de las PAM de la muestra

Se encontró que el 29.1\% de las personas de la muestra participa en un grupo, otro $29.1 \%$ participa en tres grupos, seguido por el $24.2 \%$ que participa en dos, el $7.8 \%$ en cuatro, el $4.9 \%$ en cinco, y el $4.9 \%$ en más de cinco grupos. Así, el $70.9 \%$ de las personas participantes forman parte de dos o más grupos organizados de personas mayores.

En la Tabla 1 se muestra el detalle en horas anuales de las actividades que realizan las personas participantes en los grupos organizados de personas mayores tanto en AGECO como en otras agrupaciones fuera de esa institución.

En general, se evidencia que las personas de la muestra, cuando están en los grupos, dedican mayor cantidad de horas a las actividades sociales, recreativas y físicas, aunque existen diferencias en los perfiles de las personas, pues a lo interno de todas las categorías hay gran variabilidad.

En las actividades sociales participa el mayor porcentaje de personas, el $93.2 \%$ de los casos válidos, seguidas por actividades recreativas con el $72.8 \%$, actividades físicas con el $70.6 \%$, actividades educativas con el 55.3\%, actividades artísticas con el 45.6\%, mientras las actividades que menos personas realizan

Tabla 1

Total de horas anuales en las distintas categorías de actividades grupales

\begin{tabular}{lccccc}
\hline Tipo de actividades & $N$ & $M$ & $D E$ & Valor mínimo & Valor máximo \\
\hline Físicas & 103 & 119.99 & 121.28 & 0 & 440 \\
Sociales & 103 & 103.86 & 112.34 & 0 & 660 \\
Recreativas & 103 & 85.11 & 206.53 & 0 & 1344 \\
Educativas & 103 & 73.41 & 138.55 & 0 & 832 \\
Artísticas & 103 & 63.40 & 103.10 & 0 & 520 \\
Ocio cognitivo & 103 & 43.25 & 88.92 & 0 & 520 \\
Adquisición de habilidades & 103 & 39.96 & 77.98 & 0 & 416 \\
Logísticas & 103 & 26.70 & 68.85 & 0 & 452 \\
\hline
\end{tabular}


Tabla 2

Resultados para las pruebas MMSE, Test del reloj a la orden, y a la copia, Stroop y Trail Making $A$ y $B$

\begin{tabular}{lccccc}
\hline Prueba/escala & $N$ & $M$ & $D E$ & Valor mínimo & Valor máximo \\
\hline MMSE & 109 & 28.13 & 2.10 & 19 & 30 \\
Test del reloj a la orden & 108 & 8.21 & 2.04 & 3 & 10 \\
Test del reloj a la copia & 109 & 9.15 & 1.25 & 2 & 10 \\
Stroop (Medida de inhibición de la interferencia) & 104 & 4.70 & 7.63 & -13.22 & 26.91 \\
Trail Making A (tiempo en segundos) & 108 & 73.91 & 29.35 & 20 & 208 \\
Trail Making A (Nivel de rendimiento) & 109 & 2.19 & 1.01 & 1 & 6 \\
Trail Making B (tiempo en segundos) & 102 & 177.43 & 97.69 & 40 & 573 \\
Trail Making B (Nivel de rendimiento) & 109 & 2.83 & 1.76 & 1 & 6 \\
\hline
\end{tabular}

son las actividades de ocio cognitivo con $31.1 \%$, actividades organizativas de coordinación $30.1 \%$ y finalmente actividades de adquisición de habilidades que únicamente realiza el $29.1 \%$ de las personas de la muestra. Se observa, una disminución de los porcentajes de participación en aquellas actividades que suponen una mayor demanda cognitiva, tales como las educativas, las de ocio cognitivo y las de coordinación y dirección de grupos. Es decir, un grupo reducido de personas se involucra en actividades que contribuirían de manera particular a la estimulación y mantenimiento del rendimiento cognitivo.

Así, a pesar de que las personas de la muestra no cuentan con altos ingresos ni con altos niveles de escolaridad, poseen redes sociales de apoyo, participan en actividades sociales y exhiben patrones de independencia y autonomía en su vida cotidiana y su entorno social, lo que en términos conceptuales les asociaría a los perfiles descritos de personas mayores con envejecimiento exitoso o saludable.

Descripción del rendimiento cognitivo de las Personas Adultas Mayores de la muestra

Se observó que los sujetos de la muestra evidencian un rendimiento cognitivo esperable para su edad y nivel educativo en las pruebas de cribaje del rendimiento intelectual global; mientras que en las pruebas que evalúan con mayor especificidad el funcionamiento ejecutivo y la memoria, el rendimiento es esperable normativamente, pero ubicado en los rangos medios y percentiles inferiores de calificación de las pruebas.

Para las pruebas del cribaje MMSE, Test del reloj a la orden y a la copia, las personas de la muestra obtuvieron medias cercanas a los máximos puntajes posibles cumpliendo con los criterios de inclusión. Sin embargo, se evidencia un rendimiento ubicado en los rangos y percentiles inferiores de puntaje, respecto de los puntajes máximos posibles, en las medidas que evalúan con mayor especificidad las funciones ejecutivas como la prueba Stroop y las medidas de nivel de rendimiento del test Trail making A y B; en los cuales según los criterios de evaluación de la prueba, el rendimiento promedio de la muestra se ubica en el rango "normal-bajo". Esto coincide con lo expuesto por Lowe y Rabitt (2005) en cuanto a que la proporción de enlentecimiento asociado a la edad difiere en tareas diferentes y que es consistentemente más marcado en tareas relacionadas con el funcionamiento ejecutivo, no evidenciables en las medidas generales de cribaje del funcionamiento cognitivo global.

Se puede decir que no se trata de sujetos con altos rendimientos cognitivos y ello contrasta con las descripciones internacionales de habla castellana reportadas en el estudio de caracterización de 
poblaciones adultas mayores con estilos de vida activos y envejecimiento saludable (Fernández - Ballesteros et al., 2005). Sin embargo, estas personas podrían considerarse insertas dentro de las perspectivas del envejecimiento exitoso que refieren a patrones normales, en contraposición a patrones patológicos (Baltes y Baltes, 1993). Aunque el rendimiento de las personas en las pruebas señaladas no podría considerarse cómo óptimo (término al que refieren Baltes y Baltes, 1993), les permite adaptarse a su contexto personal y social, pues se mantienen insertos en procesos de participación social y con estilos de vida activos.

En las pruebas que evalúan las funciones de memoria, el rendimiento se ubicó también en los rangos y percentiles inferiores de puntuación esperable para la edad y el nivel educativo de la muestra, en la Tabla 3 se muestran los resultados en las subescalas de la prueba CERAD. En la subescala de fluidez

Tabla 3

Resultados para la prueba CERAD

\begin{tabular}{lccccc}
\hline Tipo de actividades & $N$ & $M$ & $D E$ & Valor mínimo & Valor máximo \\
\hline CERAD Fluidez verbal & 109 & 18.76 & 4.73 & 9 & 31 \\
CERAD memoria de una lista de palabras & 109 & 6.55 & 1.64 & 2 & 10 \\
CERAD recuerdo de una lista de palabras & 109 & 4.83 & 2.07 & 0 & 9 \\
CERAD reconocimiento de una lista de palabras & 108 & 8.74 & 1.62 & 1 & 10 \\
CERAD dígitos directos & 109 & 5.39 & .99 & 3 & 7 \\
CERAD dígitos inversos & 109 & 3.30 & .94 & 2 & 5 \\
\hline
\end{tabular}

Tabla 4

Resultados para las subescalas y la escala total CAMCOG

\begin{tabular}{lccccc}
\hline Prueba/escala & $N$ & $M$ & $D E$ & Valor mínimo & Valor máximo \\
\hline Orientación temporal y espacial & 108 & 4.82 & .49 & 2 & 5 \\
Comprensión (Lenguaje) & 108 & 8.86 & .39 & 7 & 9 \\
Expresión (Lenguaje) & 108 & 17.44 & 2.06 & 12 & 21 \\
Memoria remota & 108 & 5.70 & .72 & 3 & 6 \\
Memoria reciente & 108 & 3.46 & .67 & 1 & 4 \\
Memoria aprendizaje & 108 & 12.38 & 2.37 & 5 & 17 \\
Atención y Cálculo & 108 & 8.02 & 1.43 & 2 & 9 \\
Pensamiento abstracto & 108 & 4.31 & 2.36 & 0 & 8 \\
Función ejecutiva & 108 & 16.76 & 4.51 & 7 & 26 \\
CAMCOG total & 108 & 81.77 & 10.21 & 56 & 102 \\
\hline
\end{tabular}


verbal se observa mayor variabilidad entre los sujetos y en las subescalas que evalúan memoria: "memoria de una lista de palabras" y "recuerdo de una lista de palabras" el rendimiento se ubica dentro de los rangos y percentiles inferiores de puntaje. Esto coincide con las teorías sobre el rendimiento cognitivo en patrones de envejecimiento normativo, donde se observa de manera más marcada el declive en tareas en las que intervienen las funciones ejecutivas y la memoria (Fernández - Ballesteros et al., 2005).

El rendimiento en la escala "reconocimiento de una lista de palabras" refiere a un desempeño ubicado en los rangos superiores de puntaje, respecto al puntaje máximo esperado de 10, en esta escala el puntaje que se presenta es el esperado para población normal, pues puntajes bajos indicarían deterioro cognitivo considerable. En las subescalas "dígitos directos" y "dígitos inversos" el puntaje máximo posible por alcanzar es 7 y en la prueba "dígitos inversos" que es una prueba relacionada con la memoria de trabajo los datos evidencian un rendimiento menor.

En términos generales en la escala CERAD las personas participantes muestran un rendimiento cognitivo dentro de los rangos normativos esperados para las subpruebas que miden funcionamiento cognitivo general. Mientras que en las pruebas que evalúan memoria y funciones ejecutivas, muestran un rendimiento bajo respecto del puntaje máximo posible. Estos hallazgos coinciden con lo planteado por CahnWeiner, Malloy, Boyle, Marran y Salloway (2000) y Plehn et al. (2004) quienes afirman que las medidas sensibles al funcionamiento ejecutivo y memoria son las que más contribuyen a discriminar entre el rendimiento de las personas adultas mayores y su participación social, sobre todo considerando que la memoria es una de las variables asociadas al funcionamiento social y que los cambios en el rendimiento de ésta se relacionan con cambios en la interacción social.

Los sujetos muestran un rendimiento esperable para su edad y nivel educativo en las subescalas de orientación en tiempo y espacio, en este rubro se refleja poca variabilidad entre los sujetos. También en el área de lenguaje se evidencia un alto rendimiento en la escala de "comprensión de lenguaje" que exige tanto respuestas verbales como respuestas motoras, la media fue $M=8.86, D E=.39$. Para la escala "expresión de lenguaje" en la que también interviene el rendimiento en memoria el puntaje máximo posible es 21 y la media $M=17.44, D E=2.06$, indicando un rendimiento ubicado en los rangos medios de puntaje de la prueba. Estas escalas muestran mayor variabilidad y los resultados comprueban rendimientos dentro de los rangos normativos, aunque menor rendimiento y mayor variabilidad en las funciones que tienen que ver con memoria y fluidez verbal.

En las subescalas específicas que evalúan memoria se observa un alto rendimiento en la de "memoria remota" $M=5.70, D E=.72$. Este hallazgo es consistente con la teoría que señala que el funcionamiento cognitivo relacionado con el conocimiento cultural adquirido a través de los años, se mantiene estable durante los procesos de envejecimiento normal (FernándezBallesteros et al. 2005).

Los rendimientos menores se observan para las subescalas que evalúan "memoria reciente" $M=3.46$, $D E=.67$, y "memoria de aprendizaje" $M=12.38$, $D E=2.37$. Así pues, se observa menor rendimiento y mayor variabilidad entre los sujetos en las funciones de memoria a corto plazo y memoria de trabajo, confirmando en la muestra estudiada que estas funciones aportan mayores insumos para discriminar a los sujetos dentro de muestras de personas mayores sin deterioro cognitivo (Plehn et al. 2004).

Para la escala atención y cálculo $M=8.02, D E=$ 1.43 , se evidencia un alto rendimiento de los sujetos que se corresponde con los resultados de funcionamiento cognitivo general de la muestra.

Por otra parte se observa un rendimiento ubicado en los rangos medios de puntuación en la escala "pensamiento abstracto", $M=4.31, D E=2.36$ y en la escala "función ejecutiva", que incluye ítems de pensamiento abstracto, fluidez ideacional y razonamiento visual, $M=16.76, D E=4.51$. Estas escalas son las que muestran mayor variabilidad 
entre las personas participantes, lo cual es también congruente con la postura de Fernández-Ballesteros et al. (2005) y Lowe y Rabitt (2005) sobre la presencia de cambios más marcados en las funciones ejecutivas, la velocidad de procesamiento y la memoria de trabajo relacionados con la edad.

Adicionalmente, interesa describir diferencias en el rendimiento cognitivo según el sexo y la edad de las personas participantes. Para ello, se realizaron pruebas para comparar el rendimiento en las medidas cognitivas según sexo y aunque la mayoría de la muestra $(65 \%)$ estuvo constituida por mujeres no se encontraron diferencias estadísticamente significativas entre los puntajes de hombres y mujeres.

También se analizó si existían diferencias en las medidas cognitivas según la edad. Para ello se correlacionaron las variables cognitivas con la edad. Los resultados se observan en la Tabla 5.

En las escalas Trail Making A y B, pruebas relacionadas con el funcionamiento ejecutivo, las personas más envejecidas de la muestra evidencian un menor rendimiento. En las escalas de memoria de la prueba CERAD y de la prueba CAMCOG las correlaciones son negativas, esto indica que a mayor valor en una variable disminuye la otra. Lo mismo ocurre en las subescalas del CAMCOG que evalúan función ejecutiva, y en la escala total;estas correlaciones podrían interpretarse en términos de que a mayor edad menor rendimiento en las pruebas que miden memoria -particularmente memoria de trabajo- y funciones ejecutivas.

Los resultados evidencian un mejor rendimiento de las personas más jóvenes y uno menor en el grupo de las personas con rango medio de edad y en el grupo de las más envejecidas.

En síntesis, sobre el rendimiento cognitivo de las PAM de la muestra se puede decir que está ubicado en los rangos medios y bajos de las puntuaciones máximas posibles de las pruebas que miden memoria -particularmente- de trabajo y funciones ejecutivas. Este patrón de rendimiento les permite funcionar de manera independiente y autónoma en su vida cotidiana, contar con redes sociales, participar en grupos sociales y realizar distintos tipos de actividades sociales. No se observaron diferencias significativas en el rendimiento de los hombres y las mujeres de la muestra; las personas más envejecidas presentaban un menor rendimiento cognitivo tanto en las medidas de desempeño global como en las medidas de memoria de trabajo y funciones ejecutivas.

\section{Discusión}

El estilo de vida activo y la participación social en grupos organizados que caracteriza a la muestra descrita, son considerados indicadores de éxito en las teorías del envejecimiento activo y saludable (Baltes y Baltes, 1993; Fernández-Ballesteros, Caprara \& García, 2005; Fernández Ballesteros et al., 2012; OMS 2002; Rowe \& Kahn, 1997).

Tabla 5

Correlaciones entre edad y medidas cognitivas

\begin{tabular}{lc}
\hline Medida Cognitiva & $r$ \\
\hline Test del Reloj a la orden & $-.20^{*}$ \\
Trail Making A Tiempo en segundos & $.39 * * *$ \\
Trail Making A nivel de rendimiento & $.21^{*}$ \\
Trail Making B Tiempo en segundos & $.31^{* *}$ \\
Trail Making B nivel de rendimiento & $.24^{*}$ \\
CERAD Fluidez verbal & $.37^{* * *}$ \\
CERAD memoria de una lista de palabras & $.28^{* *}$ \\
CERAD recuerdo de una lista de palabras & $.37 * * *$ \\
CERAD reconocimiento de una lista de & $-.32^{* * *}$ \\
palabras & $-.23^{* *}$ \\
CAMCOG orientación temporal & $-.26^{* *}$ \\
CAMCOG memoria reciente & $-.29 * *$ \\
CAMCOG memoria de aprendizaje & $-.29 * *$ \\
CAMCOG pensamiento abstracto & $-.28^{* *}$ \\
CAMCOG función ejecutiva & $-.34 * * *$ \\
CAMCOG total & \\
\hline$* p<.05, * * p<.01, * * * p<.001$ &
\end{tabular}


Sin embargo, la muestra del presente estudio sirve de ejemplo empírico sobre las particularidades de los procesos de envejecimiento activo o exitoso, pues parece diferenciarse de las propuestas teóricas tradicionales en varios aspectos: aunque presentan bajos ingresos económicos, bajo nivel educativo y rendimientos cognitivos ubicados en rangos y percentiles medios y bajos en funciones ejecutivas y memoria; son personas independientes y autónomas, que participan socialmente en diversas actividades fuera de su hogar.

En cuanto al rendimiento cognitivo, las medias en las pruebas de cribaje que evalúan el estado cognitivo general, evidencian que se trata de un grupo bastante homogéneo en su rendimiento y que presenta un buen estado cognitivo global dentro de los criterios normativos esperados. En este sentido, debe trabajarse en la identificación y validación de pruebas que permitan mayor discriminación en el rendimiento cognitivo de PAM sin deterioro.

Sin embargo, cuando se observan las subpruebas y subescalas que evalúan funciones específicas como el funcionamiento ejecutivo y la memoria, el rendimiento se ubica en los rangos medios e inferiores de calificación de las pruebas.

Interesa señalar que el menor rendimiento de la muestra en pruebas que miden funciones ejecutivas y memoria se asoció especialmente con las personas más envejecidas. No obstante, aunque el funcionamiento de las personas en las pruebas señaladas no podría considerarse cómo óptimo (término al que refieren Baltes \& Baltes 1993), les permite adaptarse a su contexto personal y social y participar socialmente en grupos organizados de PAM.

A nivel metodológico, se evidenció que las medidas cognitivas utilizadas muestran índices adecuados de consistencia interna y evidencias iniciales de validez convergente, por lo que se recomienda su uso de manera complementaria para profundizar en las distintas dimensiones del rendimiento cognitivo en futuras investigaciones con muestras similares. En el caso del MMSE se recomienda su uso únicamente como medida de cribaje y acompañado de pruebas específicas para la valoración detallada del estado cognitivo de PAM.

Este estudio aporta evidencia empírica sobre la variabilidad en los patrones de envejecimiento saludable, en los que deben comprenderse las particularidades culturales y contextuales. En la muestra estudiada, un patrón de rendimiento cognitivo con puntajes medios o bajos en ciertas funciones parece no ser un elemento decisivo que impida el funcionamiento independiente y autónomo, con participación activa en grupos sociales. En consecuencia, se ha de reconocer la necesidad de pensar en intervenciones diferenciadas, que brinden la posibilidad de incluir dentro del ámbito de acción institucional a muchas personas mayores en su gran variabilidad interindividual. Nuevos servicios y opciones más apropiadas para su desarrollo personal son indispensables como meta en la agenda política del envejecimiento saludable y satisfactorio.

\section{Limitaciones del estudio}

Las pruebas presentan limitaciones para discriminar el rendimiento cognitivo de PAM sin deterioro cognitivo y por otra parte no se encuentran validadas para el contexto costarricense, el presente estudio aporta evidencias iniciales en ese sentido.

Resultaría importante realizar trabajos que ofrezcan aportes al estudio de la consistencia interna, validez convergente y validez discriminante de las medidas de rendimiento cognitivo utilizadas, con el fin generar procesos de adaptación y validación y establecer puntos de corte para la población adulta mayor costarricense.

El instrumento utilizado para evaluar participación social de PAM en grupos organizados es una primera aproximación a la evaluación de este aspecto en relación con procesos de envejecimiento exitoso, por tanto es necesario el trabajo en la identificación y/o creación, de pruebas validadas para evaluar el nivel de participación social de las PAM.

\section{Posibles lineas de investigación futura}

Se sugiere incluir dentro de las muestras de estudio a PAM que participen en grupos fuera de San José, 
los cuales posiblemente cuenten con menos opciones de actividades y responden a intereses puramente recreativos, con el fin de explorar si existen diferencias en el rendimiento cognitivo entre las personas que pertenecen a estos grupos y personas que cuentan con una amplia oferta de actividades.

También resultaría necesario incluir en las muestras de estudio a personas que no participen en grupos organizados, para analizar si existen diferencias en sus perfiles de rendimiento cognitivo al compararlas con personas que participan en grupos.

\section{Referencias}

Asociación Gerontológica Costarricense. (2008). Caracterización de los grupos de la Red Nacional de Clubes de Personas Mayores. Documento interno. Sin publicar.

Baltes, P.B. \& Baltes, M. M. (1993). Successful Aging. Perspective from the Behavioral Sciences. Canada: Cambridge University Press.

Blanco, M. (2007). Factores psicosociales determinantes del envejecimiento activo o exitoso en personas adultas mayores costarricenses. Tesis para optar al grado de Magister Scientiae en Gerontología. Maestría en Gerontología, Universidad de Costa Rica, San José, Costa Rica.

Brugnolo, A., Nobili, F., Barbieri, M., Dessi, B., Ferro, A., Girtler, N. et al. (2009). The factorial structure of the Mini Mental State Examination (MMSE) in Alzheimer's disease. Archives of Gerontology and Geriatrics, 49, 180-185.

Cacho-Gutiérrez, L.J., García-García, R., ArcayaNavarro, J., Vicente-Vallardón, J.L. \& LantadaPuebla, N. (1999). Una propuesta de aplicación del test del reloj en la enfermedad de Alzheimer. Revista de Neurología, 28, 648-655.

Cahn-Weiner, D., Malloy, P.F., Boyle, P.A., Marran, M., \& Salloway, S. (2000). Prediction of functional status from neuropsychological test in community - dwelling elderly individuals. The Clinical Neurospychologist, 14, 187-195.
Fernández-Ballesteros, R., Caprara, M. \& García, C. (2005). Vivir con vitalidad-M: A european multimedia programme. Psychology in Spain, 9(1), $1-12$.

Fernández-Ballesteros, R., Molina, M.A., Schettini, R., \& Del Rey, A.L. (2012). Promoting Active Aging Through University Programs for Older Adults An Evaluation Study. GeroPsych, 25 (3), 145-154.

Fernández, X. \& Robles, A. (Cords). (2008). I Informe estado de situación de la persona adulta mayor en Costa Rica. San José, Costa Rica: Universidad de Costa Rica.

Golden, C. (1994). Stroop: Test de colores y palabras. Manual TEA publicaciones de psicología aplicada, serie menor 226. Madrid: TEA Ediciones.

Hernández, R. Fernández, C. \& Baptista, P. (2001). Metodología de la investigación. México: McGraw Hill.

Hultsch, D.F., Small, B.J., Hertzog, C. \& Dixon, R.A. (1999). Use it or lose it: engaged lifestyle as a buffer of cognitive decline in aging? Psychology and aging, 14(2), 245-263.

Instituto Nacional de Estadística y Censos (INEC), (2012). Censo 2011. Recuperado de http://www. inec.go.cr/Web/Home/GeneradorPagina.aspx

Lander, V. (2005). A New Look at Succesful Aging : Exploring a Mild-range Nursing Theory Among Older Adults in a Low-income Retirement Community. The Journal of Construction \& Testing, 15(1), 17-23.

Llinás, J., Villalta, J. \& López-Pousa, S. (1991). CAMDEX: Adaptación y validación española. Barcelona: Áncora.

Lobo, A., Ezquerra, J., Gómez, F., Sala, J. \& Selva, A. (1979). El Mini-Examen Cognoscitivo (Un test sencillo, práctico para detectar alteraciones intelectuales en pacientes médicos. Actas Luso Españolas de Neurología y Psiquiatría, VIII 3, 189-202.

Lowe, C. \& Rabbitt, P. (2005). Cognitive models of ageing and frontal lobe deficits. En P. Rabbitt (Ed.), 
Methodology of frontal and executive function (pp.38-58). Reino Unido: Psychology Press.

Mitrushina, M. \& Satz, P. (1991). Raliability and validity of the Mini- Mental State Exam in Neurologically intact elderly. Journal of clinical psychology, 47 (4) 537 543.

Morris, J., Heyman, A., Mohs, R., Hughes, J., Van Belle, G., Fillenbaun, G. et al. (1989). The Consortium to Establish a Registry for Alzheimer's Disease (CERAD). Part I. Clinical and neuropsycological assesment of Alzheimer's disease. Neurology 39 (9), 1159-1165.

Organización Mundial de la Salud. (2002). Envejecimiento Activo: un marco político. Revista Española en Geriatría y Gerontología, 37(2), 74-105.

Peña-Casanova, J., Quiñones-Úbeda, S., QuintanaAparicio, M., Aguilar, M., adenes, D., Molinuevo, J. L., et al. (2009). Spanish multicenter normative studies (Neuronorma Project): Norms for verbal span, visuospatial span, letter and number sequencing, Trail Making Test, and Symbol Digit Modalities Test. Archives of Clinical Neuropsychology, 24, 321-341.

Plehn, K., Marcopulos, B.A. \& McLain, C.A. (2004). The Relationship Between Neuropsychological Test Performance, Social Functioning, and Instrumental
Activities of Daily Living in a Sample of Rural Older Adults. The Clinical Neuropsychologist, 18(1), 101-113.

Rowe, J.W. \& Kahn, R.L. (1997). Successful Aging. The Gerontologist, 37, 433-440.

Salazar-Villanea, M. \& Blanco-Molina, M. (noviembre, 2010). Envejecimiento y desarrollo personal y social: ¿La actividad como paradigma incuestionable? Ponencia presentada en el III Congreso Internacional de Gerontología, San José, Costa Rica.

Schooler, C. \& Mulatu, M.S. (2001). The Reciprocal Effects of Leisure Time Activities and Intellectual Functioning in Older People: A Longitudinal Analysis. Psychology and Aging, 16 (3), 466-482.

Smith, E. \& Kosslyn, S. (2008). Procesos Cognitivos. Modelos y bases neurales. Madrid: Pearson Prentice Hall.

Smits, C.H., Van Rijsselt, R.J., Jonker, C. \& Hardy, D.J. (1995). Social participation and cognitive funtioning in older adults. International Journal of Geriatric Pschiquiatry, 10, 325-331.

Verna, C.P., Sloan, J., \& Guse, L. (2000). An Examination of Psychometric Properties of the Mini-Mental State Examination and the Standardized MiniMental State Examination:Implications for Clinical Practice. Applied Nursing Research, 4 (13), 209-213.

Recibido: 01 de noviembre 2013

Aceptado: 20 de febrero 2014 